\title{
Self-reciprocating radioisotope-powered cantilever
}

\author{
Hui Li and Amit Lal ${ }^{\text {a) }}$ \\ SonicMEMS Laboratory, Department of Electrical and Computer Engineering, 402 Phillips Hall, \\ Cornell University, Ithaca, New York 14853 and Department of Electrical and Computer Engineering, \\ 1415 Engineering Drive, University of Wisconsin-Madison, Madison, Wisconsin 53706 \\ James Blanchard and Douglass Henderson \\ Department of Nuclear Engineering and Engineering Physics, 1500 Engineering Drive, University of \\ Wisconsin-Madison, Madison, Wisconsin 53706
}

(Received 14 September 2001; accepted for publication 27 March 2002)

\begin{abstract}
A reciprocating cantilever utilizing emitted charges from a millicurie radioisotope thin film is presented. The actuator realizes a direct collected-charge-to-motion conversion. The reciprocation is obtained by self-timed contact between the cantilever and the radioisotope source. A static model balancing the electrostatic and mechanical forces from an equivalent circuit leads to an analytical solution useful for device characterization. Measured reciprocating periods agree with predicted values from the analytical model. A scaling analysis shows that microscale arrays of such cantilevers provide an integrated sensor and actuator platform. (C) 2002 American Institute of Physics.
\end{abstract}

[DOI: $10.1063 / 1.1479755$ ]

\section{INTRODUCTION}

Use of radioisotopes to realize nuclear batteries has been extensively researched. ${ }^{1-4}$ One principle of operation is to collect radiated charges across a capacitor. This is called direct conversion nuclear battery. ${ }^{2}$ A direct collected-charge-tomotion conversion is demonstrated in this article.

The central idea in this article is to collect the charged particles emitted from the radioisotope by a cantilever. By charge conservation, the radioisotope thin film will have opposite charges left as it radiates electrons into the cantilever. Thus an electrostatic force will be generated between the cantilever and the radioisotope thin film. The resulting force attracts the cantilever toward the source. With a suitable initial distance the cantilever eventually reaches the radioisotope and the charges are neutralized via charge transfer. Although the exact mechanisms of charge transfer can be tunneling or direct contact, the time scale of the charge transfer is much shorter than the reciprocation cycle, allowing the details to be ignored for cantilever performance analysis. As the electrostatic force is nulled, the spring force on the cantilever retracts it back to the original position and it begins to collect charges for the next cycle. Hence, the cantilever acts as a charge integrator allowing energy to be stored and converted into both mechanical and electrical forms. Based on this idea, a prototype cantilever device has been made and a satisfactory analytical model is developed.

The self-reciprocating cantilever could be used as a selfpowered electromechanical actuator. As a mechanical actuator, the released cantilever could impact a mass for locomotion, or conversion of the mechanical energy back into electricity using the mass motion inside an electromagnet. The cantilever could also periodically deflect and modulate

\footnotetext{
a) Author to whom correspondence should be addressed; electronic mail: lal@engr.wisc.edu
}

an incident optical, radio-frequency, or ultrasonic beam which could be useful for sensing physical or chemical variables that affect the self-reciprocation times. For example, the charge collection efficiency of the cantilever is affected by the conductivity of the gas and the mechanical properties of the cantilever, which can change with environmental variables such as the presence of high-molecular weight organic compounds [chem-bio warfare agents, or drastic temperature (for detecting heat sources)] or inertial loading on the device (for sensing vibration). It provides a scalable self-powered platform for not only sensing, but also providing an actuator to modulate information. The device will be operational on the same time scale as the half life of the radioisotope, which can be several decades enabling applications such as embedded sensing in civil or military structures over the useful life of the structures. Furthermore, the temperature insensitivity of the radiation might enable high and low temperature operation, which is not possible with chemical batteries in which reactions are controlled by temperature.

\section{ELECTROMECHANICAL MODEL}

Figure 1 shows the equivalent circuit of the selfreciprocating cantilever. The radioisotope source is modeled as a current source. The cantilever/source gap is modeled as a time varying capacitor. The parasitic resistor is included to model possible leakage paths for the collected charge. Several physical mechanisms may contribute to this resistance. Both naturally occurring ions, and ions created by electronic collisions between emitted particles and gas molecules, will constitute a leakage current. Furthermore, secondary electrons emitted from the cantilever due to high energy electron-substrate collisions may contribute to the leakage current with a polarity opposite to the emitted current. Charge conservation results in

$$
\alpha I-\frac{V}{R}-\varepsilon_{0} A \frac{\partial}{\partial t}\left(\frac{V}{d}\right)=0,
$$




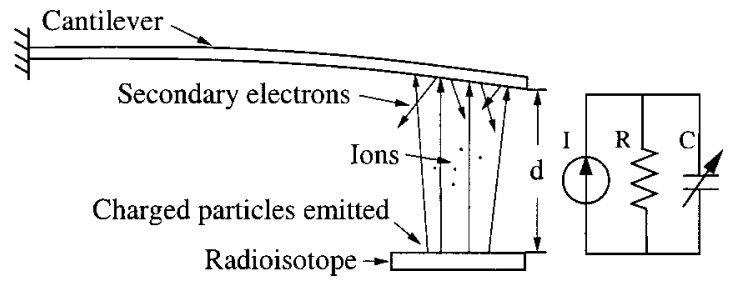

FIG. 1. Equivalent circuit of the self-reciprocating cantilever. The distance between the cantilever and the radioisotope is exaggerated. The emitted charges can be modeled as a current source. The resistor $R$ represents leakage path. The cantilever and the radioisotope form the capacitor $C$. The cantilever eventually touches the bottom electrode neutralizing the stored charges.

where $I$ is the total emitted current from the radioisotope, $A$ is the area of the capacitor, $R$ is the equivalent resistance, $V$ is the voltage across the source and the cantilever, $t$ is the time, $d$ is the distance between the electrodes, and $\alpha$ is an empirical coefficient describing the portion of the total emitted current that gets collected by the cantilever. The first term is the emitted current; the second is the leakage current and the third is the displacement current. There are at least three reasons for imperfect charge collection (i.e., $\alpha<1$ ). First, the charged particles emitted from the source have an angular distribution and only the particles that fall in the solid angle formed by the intersection of the source and cantilever are collected. Second, some high energy particles can travel through the cantilever. Third, when secondary electrons are emitted from the cantilever, positive charges are left in the cantilever, reducing the net negative charges.

The third term in Eq. (1) is the displacement current of the capacitor. The electrical field $\mathcal{E}$ between the source and the cantilever has been approximated as uniform, i.e., $\mathcal{E}$ $=V / d$, because the angle of approach between the cantilever and the source is small allowing the approximation that an average gap $d$ exists between the cantilever and the source.

Assuming that the cantilever moves very slowly, an assumption which is verified by experiment, one can ignore the cantilever's inertia. In this quasistatic approximation, the electrostatic attraction force acting on the cantilever is exactly balanced by the spring force of the cantilever. This can be written as

$$
k\left(d_{0}-d\right)=Q \mathcal{E},
$$

where $k$ is the spring constant, $d_{0}$ is the initial distance, $d$ is the distance between the cantilever and the radioisotope, $Q$ is the total charges on the cantilever and $\mathcal{E}$ is the electric field. Assuming a uniform electric field, the capacitor can be modeled as a parallel plate capacitor $C$ and the charge on it is

$$
Q=C V=\frac{\varepsilon_{0} A V}{d} .
$$

Combining Eqs. (2), (3) and with the uniform electric field approximation

$$
k\left(d_{0}-d\right)=\varepsilon_{0} A \frac{V^{2}}{d^{2}}
$$

It can be rewritten as

$$
V=\sqrt{\frac{k}{\varepsilon_{0} A}} \sqrt{d_{0}-d} d .
$$

Substituting Eq. (5) into Eq. (1) results in

$$
\frac{\partial d}{\partial t}=\frac{2}{\varepsilon_{0} R A}\left(d_{0}-d\right) d-\frac{2 \alpha I}{\sqrt{\varepsilon_{0} k A}} \sqrt{d_{0}-d} .
$$

This equation can be readily solved numerically to compare with experimental results. At atmospheric pressure, the collected charges can easily be neutralized by ionized and polarized air molecules. This will reduce $R$ to a small value, resulting in negligible $\partial d / \partial t$ as observed in experiments done in room air. To obtain large $R$, vacuum operation is required. Under the experimental conditions where the pressure is between 25 and 60 mTorr, $R$ was found to be on the order of $10^{14}-10^{15} \Omega$. This result allows the elimination of the second term in Eq. (6) and it becomes

$$
\frac{\partial d}{\partial t}=-\frac{2 \alpha I}{\sqrt{\varepsilon_{0} k A}} \sqrt{d_{0}-d},
$$

which can be solved to yield

$$
d=-\left(\alpha / t \sqrt{\varepsilon_{0} k A}+\beta\right)^{2}+d_{0},
$$

where $\beta$ is a constant of integration. Including $\beta$ in the solution implies that at $t=0$, the cantilever may not be at its original position, which models residual charges or plastic strain on the cantilever. At $t=0$ Eq. (8) leads to

$$
d_{0}-d=\beta^{2} .
$$

Denoting the residual charges as $Q_{0}$, with uniform electric field assumption, it results in

$$
\varepsilon=\frac{Q_{0}}{\varepsilon_{0} A} .
$$

Combining Eqs. (2), (9), and (10)

$$
Q_{0}=\beta \sqrt{\varepsilon_{0} k A} .
$$

Therefore, $\beta$ can be used to calculate the amount of the residual charges on the cantilever, assuming little plastic strain on the cantilever. This model can also be used to find the period of the movement. Let $d=0$ in Eq. (8), the time is the period

$$
T=\left(\sqrt{d_{0}}-\beta\right) \sqrt{\varepsilon_{0} k A} / \alpha I .
$$

In the above discussion we have not taken into account the decay of the radioisotope. Assuming that the cantilever has been working for $n$ cycles and the total time is $t_{n}$, for cycle $n+1$ Eq. (7) needs to be modified as

$$
\frac{\partial d}{\partial t}=-\frac{2 \alpha I_{0} e^{-\gamma\left(t_{n}-t\right)}}{\sqrt{\varepsilon_{0} k A}} \sqrt{d_{0}-d},
$$

where $I_{0}$ is current from the radioisotope at the very beginning and $\gamma$ is the decay constant where at time $t$ the activity of the radioisotope becomes $e^{-\gamma t}$ of the original activity. This equation results in 


$$
d=-\left[\frac{\alpha I_{0} e^{-\gamma t_{n}}}{\gamma \sqrt{\varepsilon_{0} k A}}\left(1-e^{-\gamma t}\right)+\beta\right]^{2}+d_{0} .
$$

The cycle time of cycle $n+1$ is

$$
T_{n+1}=\frac{-1}{\gamma} \ln \left(1-\frac{\gamma\left(\sqrt{d_{0}}-\beta\right) \sqrt{\varepsilon_{0} k A}}{\alpha I_{0} e^{-\gamma T n}}\right) .
$$

If the long term operation has to be considered, then Eqs. (14) and (15) can be used; otherwise Eq. (8) is sufficiently accurate for short term analysis.

\section{CHARACTERISTICS}

Many variables are important to gauge the usefulness of the cantilever. For actuator applications the force generated by the actuator is important. The force generated by the cantilever is simply the spring force, which can be calculated as $k\left(d_{0}-d\right)$. The maximum force appears when the cantilever is released and this gives

$$
F_{\max }=k d_{0} .
$$

Hence, the cantilever is able to convert nuclear energy to electrical and mechanical energy. The energy stored in the cantilever per cycle is composed of two parts. One is mechanical energy

$$
E_{M}=\frac{1}{2} k\left(d_{0}-d\right)^{2} .
$$

The other is electrical energy

$$
E_{E}=\frac{1}{2} C V^{2} .
$$

Substitute $C=\varepsilon_{0} A / d$ and Eq. (5) into the equation above

$$
E_{E}=\frac{1}{2}\left(d_{0}-d\right) d .
$$

The mechanical energy reaches its maximum $E_{\mathrm{M} \max }$ $=1 / 2 k d_{0}^{2}$ when $d$ becomes zero, i.e., when the cantilever is released. The electrical energy has its maximum $E_{\mathrm{E} \max }$ $=1 / 8 k d_{0}^{2}$ when $d=1 / 2 d_{0}$, which is found by taking $\partial E_{E} / \partial d=0$. An electromechanical coefficient $\eta$ can be defined as the ratio of the maximum mechanical energy to electrical energy. Using Eqs. (17) and (18), this coefficient can be shown to be equal to 4 . Hence,

$$
\eta=E_{\mathrm{M} \max } / E_{\mathrm{E} \max }=4 .
$$

The total energy emitted by the radioisotope in one cycle can be calculated as

$$
E_{t}=A_{c} E_{e} T,
$$

where $A_{c}$ is the activity, $E_{e}$ is the average energy of the emitted electrons and $T$ is the period. Since at the end of the cycle the electrical energy becomes zero as from Eq. (19), the energy output is the maximum mechanical energy. Therefore, the energy efficiency of the device for one cycle can be calculated as $E_{\mathrm{M} \max } / E_{t}=k d_{0}^{2} / 2 E_{t}$.

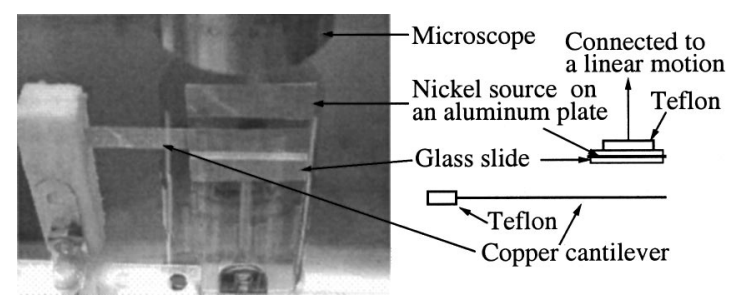

FIG. 2. A copper cantilever $(5 \mathrm{~cm} \times 5 \mathrm{~mm} \times 60 \mu \mathrm{m})$ is placed at a controlled distance away from a $1 \mathrm{mCi}^{63} \mathrm{Ni}(4 \mathrm{~mm} \times 4 \mathrm{~mm})$ electroplated on a 1-mm-thick aluminum plate.

The voltage across the cantilever and the radioisotope can be determined by Eq. (5). It is interesting to notice that the voltage has a maximum $V_{m}=\sqrt{4 k d_{0}^{3} / 27 \varepsilon_{0} A}$ and it appears when $d(t)=d_{V_{m}}=\frac{2}{3} d_{0}$.

\section{PROTOTYPE DEVICE}

A prototype device has been made to verify the cantilever operation and the model. Figure 2 shows the experimental setup. A $\beta$ source made of ${ }^{63} \mathrm{Ni}$ is used as the radioisotope. The half life of ${ }^{63} \mathrm{Ni}$ is 100.2 years. ${ }^{5}$ The $\beta$ particles (electrons) emitted have an average energy $17.3 \mathrm{KeV}$ and maximum energy $67 \mathrm{KeV} .{ }^{5}$ The ${ }^{63} \mathrm{Ni}$ is electroplated as a $4 \mathrm{~mm} \times 4 \mathrm{~mm}$ thin film on a 1 -mm-thick Al plate and the activity is $1 \mathrm{mCi}$. The cantilever is made of copper with dimensions $5 \mathrm{~cm} \times 4 \mathrm{~mm} \times 60 \mu \mathrm{m}$. The thickness $60 \mu \mathrm{m}$ was chosen to capture most of the electrons as the penetration depth of a $67 \mathrm{KeV}$ electron in copper is approximately 14 $\mu \mathrm{m} .{ }^{6}$

The cantilever is clamped between two nonconductive Teflon blocks for electrical insulation. The source is clamped by two glass slides, which are mounted on a Teflon base. The Teflon base is in turn mounted on a linear motion stage used to control the initial distance between the source and the cantilever. The setup is placed inside a vacuum chamber with a glass top. A microscope connected to a charge coupled device (CCD) camera outside the chamber is used to the monitor the gap between the source and the cantilever.

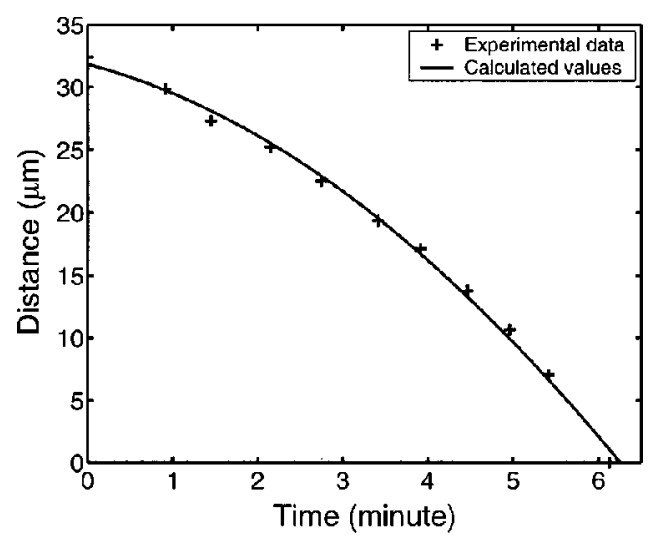

FIG. 3. Measured and calculated distance vs time curves for the experimental cantilever actuated by the ${ }^{63} \mathrm{Ni}$ source. The initial gap is $32 \mu \mathrm{m}$. 


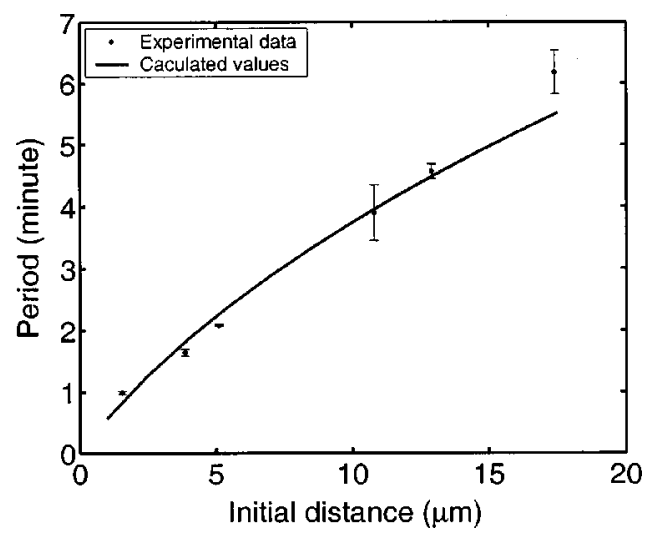

FIG. 4. Measured and calculated periods for the self-reciprocating cantilever with different initial gaps.

\section{EXPERIMENTAL RESULTS}

The experiment results verify our idea. Figure 3 shows the distance versus time for an initial distance of $32 \mu \mathrm{m}$ with a period of $6 \mathrm{~min}$ and $8 \mathrm{~s}$. In one of the experiments, the cantilever has been reciprocating continuously for four weeks, the experiment stopped by the need to use the experimental setup for other experiments. The distance versus time data were used to extract $\alpha$ and $\beta$. Parameters $k, A, I$ were determined from the properties of the materials and the device dimensions. The distance profile predicted by the model has a good fit with the data.

Figure 4 shows the measured period as a function of the initial gap along with the calculated results for fixed $\alpha$ and $\beta$. The small value of $\beta$ corresponds to approximately 2.3 $\times 10^{-11} \mathrm{C}$ charges. Although $\alpha$ and $\beta$ are different for each initial distance, the good fit between measured periods and theory for fixed $\alpha$ and $\beta$ shows the relative invariance in the gap regime of 1-15 $\mu \mathrm{m}$. The values of $\alpha$ and $\beta$ and their variations can be further used to investigate the contact process.

The energies stored in the cantilever and the voltage across the cantilever and the source are plotted in Figs. 5 and 6 , respectively. The maximum mechanical energy is $0.15 \mathrm{~nJ}$ and maximum electrical energy is $37.5 \mathrm{pJ}$. The average mechanical power can be calculated by dividing the total energy by the reciprocation period yielding $0.4 \mathrm{pW}$. At the same

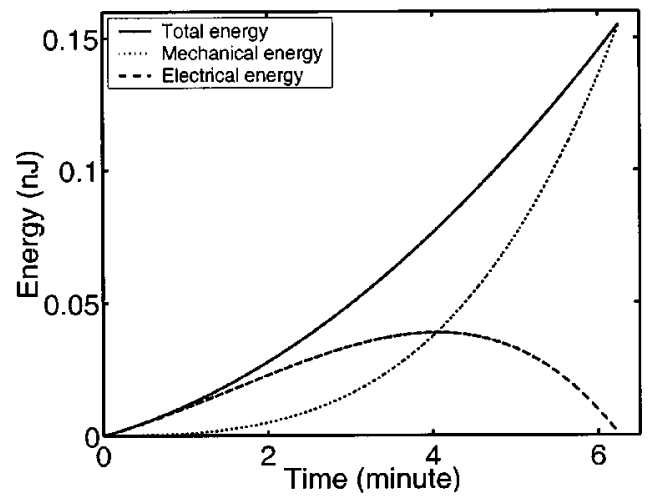

FIG. 5. Energies stored in the cantilever are plotted against time with an initial gap of $32 \mu \mathrm{m}$ for the experimental cantilever.

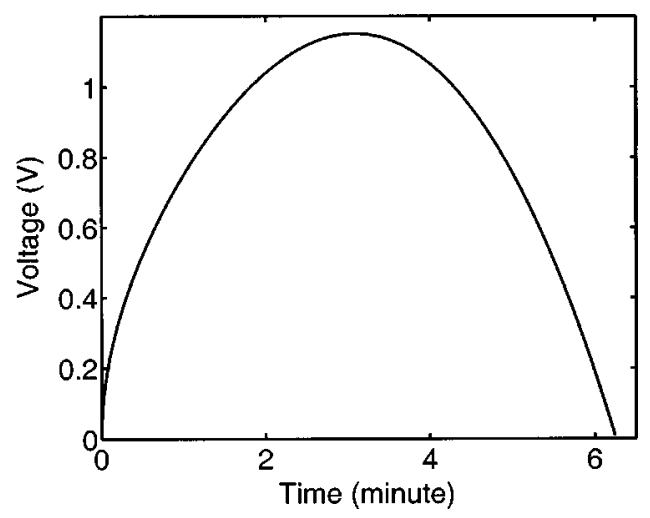

FIG. 6. The calculated voltage across the experimental cantilever and the radioisotope is plotted against time with an initial gap of $32 \mu \mathrm{m}$.

time the spring force of the cantilever reaches the maximum and it is $10.1 \mu \mathrm{V}$. The maximum voltage is $1.1 \mathrm{~V}$. The total energy from the radioisotope during this cycle is 3.8 $\times 10^{4} \mathrm{~nJ}$. So the efficiency of the cantilever is $4 \times 10^{-6}$. Although the efficiency of the cantilever is very low, it can operate for at least as long as the half life of the radioisotope, which for ${ }^{63} \mathrm{Ni}$ is $100 \mathrm{yrs}$. Assuming the operational lifetime of the prototype cantilever is 100 yrs., the total energy output would be $0.91 \mathrm{~mJ}$. This leads to a volume energy density of $5.0 \times 10^{-2} \mathrm{~J} / \mathrm{cm}^{3}$.

\section{DISCUSSION}

The radioisotope thin film is the source of the current which is important for determining the reciprocation period, as shown by Eqs. (12) and (15). Given the tremendous number of radioisotopes available, the properties that make one better than another need to be investigated. In general, radioisotopes emit alpha, beta particles and gamma rays. To avoid possible radioactive hazard with high energy alpha particles and deep penetrating gamma rays, we concentrate on radioisotopes that only emit low energy beta particles. Table I shows some candidate beta emitters with their half life and specific activity. ${ }^{5}$ The half life determines the useful length of time over which the described self-reciprocating actuator will work. Short lifetime sources are probably not important as chemical batteries (e.g., lithium or other primary cells) could effectively compete except for applications requiring high-temperature operation. However, long lifetime sources provide functionality over a period which is likely to be much larger than the shelf life of chemical batteries. Another parameter to qualify the source is the specific activity, which indicates the amount of mass needed to achieve a given activity. The smaller the specific activity, the larger the possible activity for a fixed mass. A related measure is the activity volume density listed as the last column in Table I. Since deposition of source thin films will normally be in microns of dimensions, we can use the activity volume density to quickly estimate possible maximum activity. From the table, ${ }^{32} \mathrm{P}$ has the highest volume density, however the short half lifetime makes ${ }^{32} \mathrm{P}$ less attractive. Another figure of merit is the penetration depth of the electrons into the cantilever material, which is tabulated as the last column in Table I. The 
TABLE I. Candidate beta emitting radioisotopes. The first three columns are obtained from Ref. 5, while the activity volume density is derived from the specific activity. The last column is the estimated range of electron penetration in copper (see Ref. 6).

\begin{tabular}{cccccc}
\hline \hline Radioisotope & $\begin{array}{c}\text { Average energy } \\
(\mathrm{KeV})\end{array}$ & $\begin{array}{c}\text { Half life } \\
(\text { Year })\end{array}$ & $\begin{array}{c}\text { Specific activity } \\
(\mathrm{g} / \mathrm{mCi})\end{array}$ & $\begin{array}{c}\text { Activity } \\
\text { Volume density }\left(\mathrm{mCi} / \mu \mathrm{m}^{3}\right)\end{array}$ & $\begin{array}{c}\text { Estimated range } \\
\text { in copper }(\mu \mathrm{m})\end{array}$ \\
\hline${ }^{63} \mathrm{Ni}$ & 17.4 & 100.2 & $1.76 \times 10^{-5}$ & $5.06 \times 10^{-7}$ & 14 \\
${ }^{32} \mathrm{Si}$ & 68.8 & 172.1 & $1.54 \times 10^{-5}$ & $1.51 \times 10^{-7}$ & 107 \\
${ }^{90} \mathrm{Sr}$ & 195.8 & 28.8 & $7.25 \times 10^{-6}$ & $3.50 \times 10^{-7}$ & 332 \\
${ }^{106} \mathrm{Ru}$ & 10.03 & 1.06 & $3.03 \times 10^{-7}$ & $4.08 \times 10^{-5}$ & 5 \\
${ }^{32} \mathrm{P}$ & 694.9 & 0.04 & $3.50 \times 10^{-9}$ & $5.20 \times 10^{-4}$ & 1344 \\
\hline \hline
\end{tabular}

range was calculated by using the continuously slowing down approximation range data from Ref. 6. For optimum charge capture, one would like to choose a material with a small absorption depth or isotopes with low electron energy. According to the table, nickel and ruthenium are good candidates for thin cantilevers. In this article we concentrated on ${ }^{63} \mathrm{Ni}$ as it is easily electroplated and is commonly used in MEMS fabrication.

Figure 7 shows the calculated reciprocation periods for the same cantilever used in the experiments with different radioisotopes. The activity of the source is $1 \%$ of the maximum possible activity calculated from the specific activity so that it is comparable to the ${ }^{63} \mathrm{Ni}$ source used in the experiment. One observation from the plot is that by using a suitable radioisotope a shorter or longer period of time can be achieved. On the other hand, for a given radioisotope, one can calculate the reciprocation period change with time. This curve can be used to estimate the useful lifetime of the devices.

Device scaling is another important consideration. Equation (12) exposes the possibility of scaling down the size of the device. Assuming that the electrostatic force is applied at the very end of the cantilever, the spring constant of the cantilever can be calculated as

$$
k=E w h^{3} / 4 l^{3},
$$

where $h$ is the thickness of the cantilever, $w$ is the width, $l$ is the length and $E$ is the Young's modulus. As the parallel plate

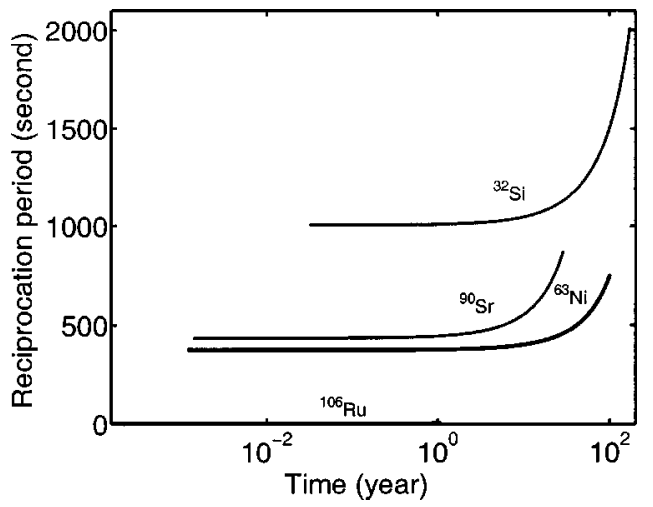

FIG. 7. Reciprocation period vs time for some beta emitting radioisotopes. As the radioisotope decays, the period changes. The periods for ${ }^{106} \mathrm{Ru}$ are in the range of $4.8-9.6 \mathrm{~s}$ placing the curve close to the bottom axis. assumption has been used before, where the source has the same width as the cantilever, the current from the source can be written as

$$
I=i A=i w L_{a},
$$

where $i$ is the current per unit area from the source, $w$ is the width, $L_{a}$ is the length of the source and $A$ is the same as in Eq. (12). Substituting Eqs. (22) and (23) into Eq. (12) and neglecting $\beta$, which can be made small, one gets:

$$
T=\sqrt{\varepsilon_{0} E d_{0} h^{3} / 4 l^{3} L_{a}} / \alpha i .
$$

Therefore, once the Young's modulus of the beam material $(E)$ and the radioisotope $(i)$ are determined, the design of the cantilever will focus on choosing the thickness and width of the cantilever and the length of the source so that for a given initial distance a desired period is obtained. A MEMS version of the cantilever is feasible according to this argument. For example, a Si cantilever with dimensions $500 \mu \mathrm{m}$ $\times 100 \mu \mathrm{m} \times 2 \mu \mathrm{m}$ can finish one cycle in about 8 min with a $200 \mu \mathrm{m} \times 100 \mu \mathrm{m}{ }^{63} \mathrm{Ni}$ source for an initial distance of 2 $\mu \mathrm{m}$. This is assuming the same unit area activity (0.06 $\mathrm{mCi} / \mathrm{mm}^{2}$ ) used in the experiment in this article. One issue is that $2-\mu \mathrm{m}$-thick silicon is not thick enough to capture most of the electrons. A solution to it is to electroplate a metal layer at the tip of the cantilever that is thick enough for electron capture. The added mass should not affect the spring constant much since the metal layer is placed at a location of low strain. If the per unit area activity is increased by a factor of 10 , which is possible given the higher specific activity, and the spring constant is decreased by reducing polysilicon thickness or increasing length, one should get much shorter reciprocation. For example, a beam $1 \mu \mathrm{m}$ thick, $1 \mathrm{~mm}$ length and $100 \mu \mathrm{m}$ width, using a source with a unit area activity of $0.6 \mathrm{mCi} / \mathrm{mm}^{2}$ and $200 \mu \mathrm{m} \times 100 \mu \mathrm{m}$ size, and assuming a better charge collection efficiency of 50\% $(\alpha=0.5)$, one should obtain a reciprocation period of $1.1 \mathrm{~s}$. Even though the total mechanical energy is only $19 \mathrm{fJ}$, it is important to remember that the same energy would be required to move this cantilever by any other means.

To increase mechanical energy one would need to increase the effective integrating time of the charge collection by increasing the initial gap $d_{0}$. Hence a trade-off between reciprocating time and stored energy exists, and the application will determine the choices. For example, in optical beam modulation applications, energy required is very small and smaller reciprocation times will be possible for high band- 
width modulation. However, for applications requiring conversion of mechanical to electrical energy, higher energies are required at the cost of longer reciprocation times.

\section{CONCLUSION}

An electrostatic cantilever actuated by radioisotopeemitted electrons has been demonstrated. A satisfactory electromechanical model that can be used to optimize the actuator performance has been developed. To meet therequirement of either signal modulation or power conversion applications, one can design the cantilever system by choosing different radioisotopes, different materials and their dimensions. Scaling the size down to MEMS scale is feasible according to the analysis, which might enable miniature self-powered arrays of cantilevers on one substrate. The long half life of the source enables the cantilever to be used as an electromechanical transducer for applications requiring long time op- eration. Furthermore, the temperature insensitivity of the radioisotope charge particle emission might enable extreme high or low temperature operation, not possible with chemical batteries.

\section{ACKNOWLEDGMENTS}

This work is supported by the U.S. DOE under NEER Grant No. DE-FG07-99ID13781 and DARPA-MTO Grant No. DAAH01-01-1-R003.

\footnotetext{
${ }^{1}$ A. Thomas, Nucleonics 13, 129 (1955).

${ }^{2}$ J. H. Coleman, Nucleonics 11, 42 (1953).

${ }^{3}$ E. G. Linder and S. M. Christian, J. Appl. Phys. 23, 1213 (1952).

${ }^{4}$ J. Braun, L. Fermvik, and Å. Stenbäck, J. Phys. E 6, 727 (1973).

${ }^{5}$ G. Harder, Pocket Guide for Radiological Management (Perma-Fix Environmental Services, 1999).

${ }^{6}$ Stopping Powers for Electrons and Positrons, International Commission on Radiation Units and Measurements, 1984.
} 